\title{
Genomic Insights into Nematicidal Activity of a Bacterial Endophyte, Raoultella ornithinolytica MG against Pine Wilt Nematode
}

\author{
Gnanendra Shanmugam ${ }^{1}$, Akanksha Dubey ${ }^{1}$, Lakshmi Narayanan Ponpandian ${ }^{1}$, Soon Ok Rim ${ }^{1}$, \\ Sang-Tae $\mathrm{Seo}^{2}$, Hanhong Bae ${ }^{1 *}$, and Junhyun Jeon ${ }^{1 *}$ \\ ${ }^{1}$ Department of Biotechnology, Yeungnam University, Gyeongsan, Gyeongbuk 38541, Korea \\ ${ }^{2}$ Division of Forest Insect Pests and Diseases, National Institute of Forest Science Seoul 02455, Korea
}

(Received on October 20, 2017; Revised on March 9, 2018; Accepted on March 11, 2018)

Pine wilt disease, caused by the nematode Bursaphelenchus xylophilus, is one of the most devastating conifer diseases decimating several species of pine trees on a global scale. Here, we report the draft genome of Raoultella ornithinolytica MG, which is isolated from mountain-cultivated ginseng plant as an bacterial endophyte and shows nematicidal activity against $B$. $x y$ lophilus. Our analysis of $R$. ornithinolytica MG genome showed that it possesses many genes encoding potential nematicidal factors in addition to some secondary metabolite biosynthetic gene clusters that may contribute to the observed nematicidal activity of the strain. Furthermore, the genome was lacking key components of avermectin gene cluster, suggesting that nematicidal activity of the bacterium is not likely due to the famous anthelmintic agent of wide-spread use, avermectin. This genomic information of $\boldsymbol{R}$. ornithinolytica will provide basis for identification and engineering of genes and their products toward control of pine wilt disease.

Keywords : bacterial endophyte, nematicidal activity, pine wilt disease (PWD)

\section{Handling Associate Editor : Seo, Young-Su}

\footnotetext{
*Co-Corresponding authors.

$\mathrm{HBae}$

Phone) +82-53-810-3031, FAX) +82-53-810-4769

E-mail) hanhongbae@ynu.ac.kr

J Jeon

Phone) +82-53-810-3030, FAX) +82-53-810-4769

E-mail)jjeon@yu.ac.kr

(c) This is an Open Access article distributed under the terms of the Creative Commons Attribution Non-Commercial License (http:// creativecommons.org/licenses/by-nc/4.0) which permits unrestricted noncommercial use, distribution, and reproduction in any medium, provided the original work is properly cited.
}

Articles can be freely viewed online at www.ppjonline.org.
Bursaphelenchus xylophilus, commonly known as pine wood nematode (PWN) is a causal agent of pine wilt disease (PWD) that causes extensive damage to forest ecosystem. The nematodes uses Monochamus beetles as vectors to infect mainly Pinus species and causes xylem dysfunction in their host, resulting in fatal wilting symptom (Mota and Vieira, 2008). In general, controls of PWD rely on aerial application of insecticides to prevent vector transmission. In addition, injection of chemicals, such as broad-spectrum of nematicides including avermectin and abamectin into the tree trunk is widely put in practice (Lee et al., 2003; Takai et al., 2003). Use of synthetic pesticides and chemicals raises great environmental concerns towards non-target organisms and human health (Jayaraj et al., 2016). In line with this, the nematicidal activities of bacteria have been regarded as alternative sources of ecofriendly biological controls of PWN (Eljounaidi et al., 2016; Zheng et al., 2016a).

Raoultella ornithinolytica strain MG ( $R$. ornithinolytica MG hereafter) is a gram negative bacterium recently isolated from mountain-cultivated ginseng (MG) plants in South Korea (Khan et al., 2017). We found that ethyl acetate extract (EtOAc) of $R$. ornithinolytica MG culture has nematicidal activity against PWNs. To identify and understand potential genome-encoded factors that are associated with the nematicidal activity of this bacterium, here we set out to determine the draft genome of $R$. ornithinolytica MG. Furthermore, we predicted genes involved in production of compounds including secondary metabolites, which are potentially contributing to nematicidal activity.

The $R$. ornithinolytica MG strain was identified based on morphological attributes and $16 \mathrm{~S}$ rDNA sequencing in our previous study (Khan et al., 2017). R. ornithinolytica MG strain was deposited in the Korean Collection for Type Cultures (deposition number: KCTC13338BP). The ge- 
nomic DNA of $R$. ornithinolytica $\mathrm{MG}$ was extracted from an overnight bacterial culture grown at $37^{\circ} \mathrm{C}$ at $200 \mathrm{rpm}$ in nutrient broth [peptone $0.5 \% \mathrm{w} / \mathrm{v}$ (Becton, Dickinson and Company, Franklin Lakes, NJ, USA), yeast extract $0.5 \%$ w/v (Duchefa Biochemie, Haarlem, The Netherlands), agar $0.5 \% \mathrm{w} / \mathrm{v}$ (Becton, Dickinson and Company), $\mathrm{NaCl} 0.5 \%$ w/v; pH 6.8]. Standard phenol-chloroform method was used for genomic DNA extraction (He, 2011; Maniatis et al., 1982).

The $500 \mu \mathrm{l}$ of $R$. ornithinolytica MG culture grown for $12 \mathrm{~h}$ was transferred to the $500 \mathrm{ml}$ of Tryptic Soy Broth (TSB) (Becton, Dickinson and Company) in $1000 \mathrm{ml}$ Erlenmeyer flask and incubated for 5 days at $30^{\circ} \mathrm{C}$ with 150 rpm. After 5 days, an equal volume of EtOAc $(500 \mathrm{ml})$ (Duksan Reagents, Ansan, Korea) was added to the broth culture and mixed well by shaking vigorously, followed by sonication for $30 \mathrm{~min}$. The EtOAc mixture was incubated for overnight on orbital shaker at $130 \mathrm{rpm}$ and allowed to stand for $2 \mathrm{~h}$. The top clear phase was transferred to a round flask and condensed in a rotary evaporator at $40^{\circ} \mathrm{C}$ (Paul et al., 2013). The crude EtOAc extract was dissolved in a solution of 3\% Dimethyl sulfoxide (DMSO) (Junsei, Tokyo, Japan) and then dried completely. Approximately 100 B. xylophilus nematodes (mixture of L2, L3 and L4/ adult stages) were taken in $90 \mu 1$ of autoclaved distilled water in each well and the nematicidal activity of total metabolites of EtOAc extracts were tested by adding $10 \mu \mathrm{l}$ at various concentrations (1000 ppm to $100 \mathrm{ppm}$ ) with six technical replications per concentration in a 96-well plate on different days, and 3\% DMSO was used as control. The plates were then incubated for three days at $20^{\circ} \mathrm{C}$ and the numbers of alive/dead nematodes were counted at 12, 24 and $48 \mathrm{~h}$ under a microscope and the percentage of nematode mortality was calculated (Choi et al., 2006; Guo et al., 2017).

Genome sequencing was performed with a PacBioRS II (Pacific Biosciences, Menlo Park, California, USA) at Theragen Etex Co., Ltd (Suwon, Korea). Raw sequences were assembled using the hierarchical genome assembly process (HGAP3) de novo assembler (Chin et al., 2013), and the resulting genome was annotated by NCBI prokaryotic genome automatic annotation pipeline (PGAAP) (Tatusova et al., 2016). The draft genome sequence of $R$. ornithinolytica strain MG was deposited in GenBank under the accession number $\mathrm{CP} 017802$.

Secondary metabolite gene clusters in the $R$. ornithinolytica $\mathrm{MG}$ genome were predicted using anti-SMASH search (Weber et al., 2015). Comparative analysis of $R$. ornithinolytica strains were performed and visualized with CLC Genomics Workbench (v9.0.1) (QIAGEN, Aarhus, Denmark, http://www.clcbio.com).

Ethyl acetate extract of the bacterial culture was tested for its potential nematicidal activity by measuring proportion of nematodes that are killed by addition of varying concentration of extract. Compared to the control (3\% DMSO), the extract showed about $40 \%$ nematicidal activity at concentrations ranging from 10 to 70 ppm (Fig. 1). Nematicidal activity started to increase at higher concentration (over $100 \mathrm{ppm}$ ) and peaked at 1000 ppm nearly annihilating $B$. xylophilus population under treatment. These results clearly suggest the potential of $R$. ornithinolytica MG as a source of compounds having activity against $B$. xylophilus.

Given such activity of $R$. ornithinolytica $\mathrm{MG}$ against

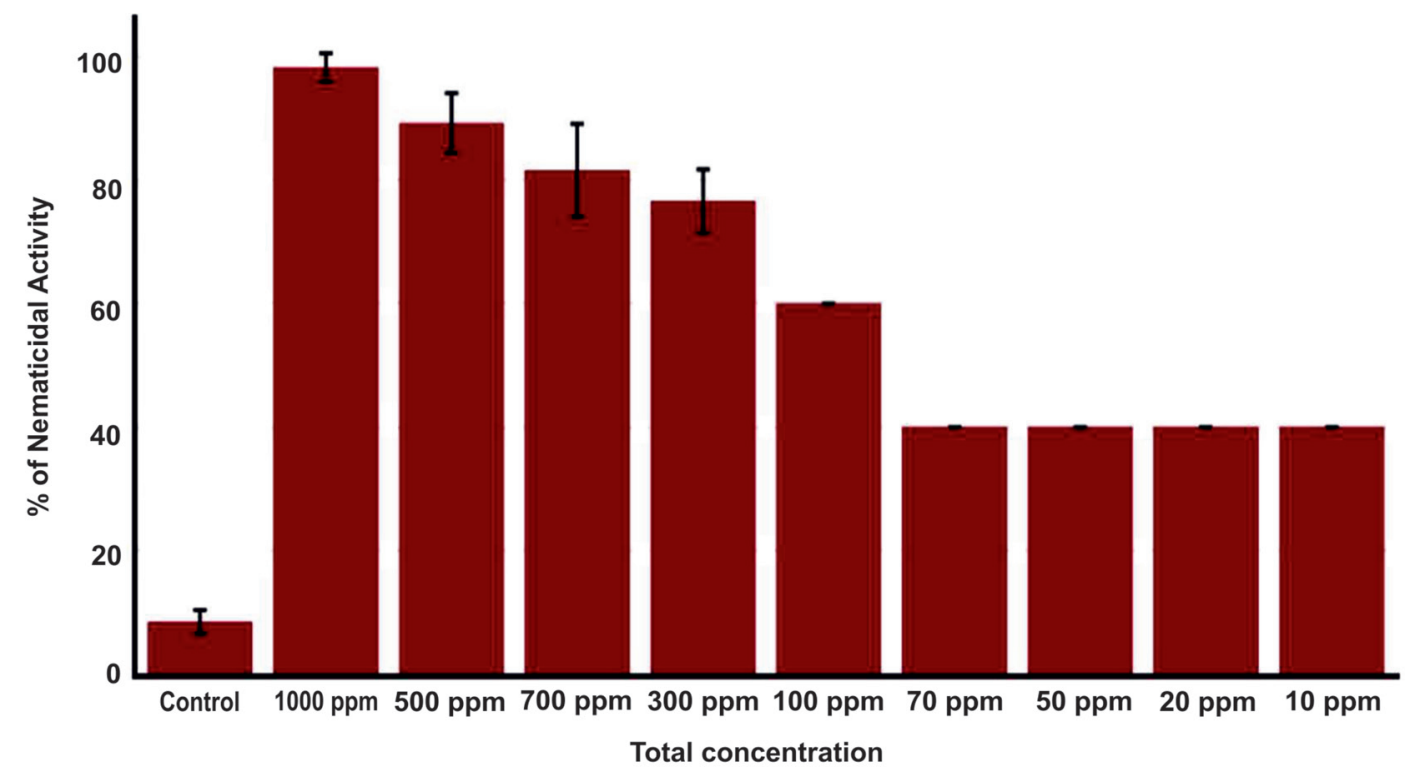

Fig. 1. Nematicidal activity of Raoultella ornithinolytica MG secondary metabolites against Bursaphelenchus xylophilus. Nematicidal activity was measured as percentage of dead Bursaphelenchus xylophilus nematodes (y-axis) over different concentrations (ppm) of $R$. ornithinolytica $\mathrm{MG}$ secondary metabolites (xaxis) with reference to $3 \%$ DMSO as control. 
Table 1. Annotated genomic features of Raoultella ornithinolytica strain MG

\begin{tabular}{lc}
\hline \multicolumn{1}{c}{ Features } & Numbers \\
\hline Genbank accession & CP017802 \\
Genome size $(\mathrm{bp})$ & $5,719,363$ \\
G+C content $(\%)$ & 55.67 \\
Genes (coding) & 5132 \\
rRNA & 25 \\
tRNA & 85 \\
ncRNA & 18 \\
Pseudogenes & 62 \\
\hline
\end{tabular}

B. xylophilus, we carried out sequencing of bacterial draft genome to provide genomic basis for pinpointing genetic components that endow the bacteria with nematicidal activity. Subsequently, a total of $1,066,832,850$ bp were sequenced in 114,707 reads, which were assembled using the hierarchical genome assembly process (HGAP3) de novo assembler, resulting in average coverage depth of $182 \mathrm{x}$. This final assembly of the entire $R$. ornithinolytica MG was determined to be $5,719,363$ bp with $\mathrm{G}+\mathrm{C}$ content of $55.67 \%$ with 3 contigs. Among the contigs, the largest one was 5,499,520 bp long, accounting for about $96 \%$ of entire genome and the remaining contigs were $218,906 \mathrm{bp}$ and 937 bp long, respectively.

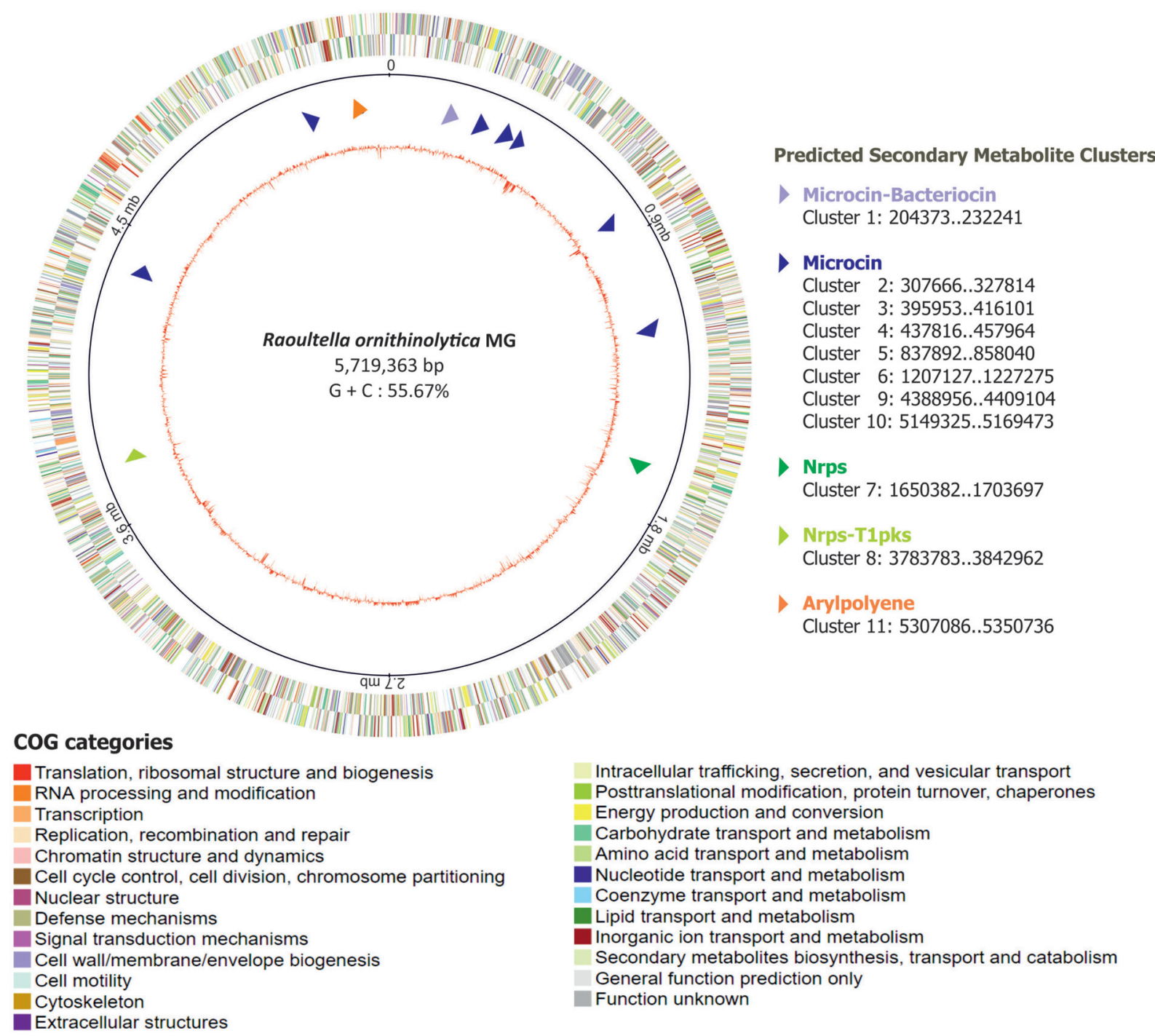

Fig. 2. Genome map of the $R$. ornithinolytica MG chromosome showing the largest contig (contig 1, which accounts for $96 \%$ of draft genome). The circular genome map with genes present on plus strand and minus strands is shown as outer and inner rings, respectively, with COG category coloring scheme. The predicted secondary metabolites gene clusters (11 cluster) along with their start and end regions were shown in arrow representations. The GC percentage along the genome map is represented as inner most circle in red. 
A total of 5,322 genes in the draft genome of $R$. ornithinolytica MG was annotated by NCBI Prokaryotic Genome Automatic Annotation Pipeline (PGAAP). These includes 5,132 coding genes, 128 RNA genes and 62 pseudogenes (Table 1). Among 5,132 coding genes, 4,445 coding genes (86.61\%) were assigned putative functions based on functional annotation. The subsystem features were annotated by RAST server (Aziz et al., 2008) and visualized by using $\mathrm{CiVi}$ web based visualization tool (Overmars et al., 2015) (Fig. 2).

In comparison, the complete genome of $R$. ornithinolyti$c a$ strain B6 (Shin et al., 2013) from public database (NCBI Accession: CP004142) is composed of a circular chromosome of $5,398,151$ bp with $55.9 \%$ GC content. A total of 4,909 coding genes, 79 tRNAs, and 25 rRNAs were annotated, and 4,070 coding genes $(82.90 \%)$ were predicted to have putative functions. This implies that the $R$. ornithinolytica strain MG possesses larger genome encoding more number of genes than strain B6.

In order to mine the nematicidal factors in $R$. ornithinolytica $\mathrm{MG}$, we took two approaches. In the first approach, we attempted to predict and catalogue gene clusters that are involved in secondary metabolite biosynthesis, potentially contributing to nematicidal activity against $B$. xylophilus. The anti-SMASH search for secondary metabolite biosynthesis gene clusters on the genome of $R$. ornithinolytica MG showed that there are 11 biosynthetic gene clusters related to the production of different types of secondary metabolites including Microcin (7 clusters), Microcin-Bacterocin (1 cluster), Nrps (1 cluster), Nrps-T1PKS (1 cluster), and Arylpolyene (1 cluster) (Table 2, Supplementary Fig. 1, $2)$. The presence of these gene clusters was also observed in the genomes of $R$. ornithinolytica strains B6 (2,3-Butanediol-producing bacterium isolated from oil-contaminat- ed soil) (NCBI Accession: CP004142) (Shin et al., 2013), $R$. ornithinolytica strain S12 (Lignin-degrading bacterium isolated from forest soil) (NCBI Accession: CP010557) (Bao et al., 2015) and A14 (Isolated from animal feces) (Leung et al., 2016) (NCBI Accession: CP008886.1) (Table 2). Interestingly, only three gene clusters such as Nrps (1 cluster), Arylpolyene (1 cluster) and Bacteriocin (1 cluster) were found on the genome of $R$. ornithinolytica strain $\mathrm{S} 12$, while the remaining two isolates contained the 11 clusters. In general, Microcin and Bacteriocin mimics siderophore so as to target and eliminate the enteric pathogens. The Arylpolyene gene clusters are similar to carotenoids. The Nrps cluster genes are known for their biological activities and pharmacological properties (Agrawal et al., 2016).

In the second approach, we examined presence/absence of 42 known nematicidal factors (Zheng et al., 2016b) in the $R$. ornithinolytica MG genome using BLASTP search (Altschul et al., 1990) (Supplementary Table 1). This search revealed presence of 9 potential nematicidal factors encoded in the genome. These include homologs of amidophosphoribosyltransferase (Xia et al., 2011), Calciumtransporting ATPase (Fan et al., 2007), Chitinase (including basic and endochitinases) and Serrawettin W2 (Fragment) (Zheng et al., 2016b) (Table 3).

To rule out the possibility that nematicidal activity of $R$. ornithinolytica $\mathrm{MG}$ is mediated by avermectin, we searched the genome for orthologous genes in avermectin (anti-nematode agent) biosynthetic gene (18 genes) (Kim et al., 2016). This showed that 5 key genes including AveR (Transcriptional regulator), AveC (Modification: spiroketal moiety C22-23 dehydration), AveE (Modification: Furan ring reduction), AveBVI and AveBVII (Modification: Addition of sugar moiety) are missing (Ikeda et al., 1999) (Supplementary Fig. 2), suggesting that the observed ne-

Table 2. Predicted secondary metabolite biosynthesis gene clusters on the genome of Raoultella ornithinolytica MG

\begin{tabular}{ccccc}
\hline \multirow{2}{*}{$\begin{array}{c}\text { Biosynthetic gene } \\
\text { clusters }\end{array}$} & \multicolumn{3}{c}{ R. ornithinolytica } \\
\cline { 2 - 4 } Cluster1 & Strain MG & Strain B6 & Strain S12 & Strain A14 \\
Microcin-Bacteriocin & Nrps-T1pks & Arylpolyene & Microcin \\
Cluster2 & Microcin & Nrps & Bacteriocin & Microcin \\
Cluster4 & Microcin & Microcin & Microcin \\
Cluster5 & Microcin & Microcin & Mrocin \\
Cluster6 & Microcin & Microcin & Nrps-T1pks \\
Cluster7 & Microcin & Microcin & Microcin \\
Cluster8 & Nrps & Microcin & Microcin \\
Cluster9 & Nrps-T1pks & Microcin-Bacteriocin & & Arylpolyene \\
Cluster10 & Microcin & Arylpolyene & & Microcin-Bacteriocin \\
Cluster11 & Microcin & Microcin & Microcin \\
\hline
\end{tabular}


Table 3. Predicted nematicidal factors homolog on the genome of Raoultella ornithinolytica MG $(E$-value $<0.001)$

\begin{tabular}{lcc}
\hline \multicolumn{1}{c}{ Known nematicidal factor (Uniprot ID) } & Homolog in R. ornithinolytica MG & $E$-Value \\
\hline Amidophosphoribosyltransferase (P00497) & WP_015583773.1 & 2e-104 \\
& WP_015585459.1 & 2-16 \\
Calcium-transporting ATPase (Q5KCV6) & WP_004863810.1 & 2e-159 \\
& WP_004859205.1 & WP_015585300.1 \\
& WP_015585008.1 & WP_015584876.1 \\
Chitinase (Q5MNU2, A9LI60, A6Y9S8, B5L0W0, A9Q0I9 and Q8TF88) & WP_004860814.1 & 5e-13 \\
Serrawettin W2 (Fragment) (K7WRE4) & WP_004862380.1 & 3e-27 \\
\hline
\end{tabular}

maticidal activity of the bacterium cannot be attributed to avermectin.

Although genome sequences of a few $R$. ornithinolytica strains including the strain B6 were deposited in NCBI, the genome sequence of $R$. ornithinolytica $\mathrm{MG}$ is the first to be reported with nematicidal activity against $B$. xylophilus. Considering the nematicidal activity, the availability of $R$. ornithinolytica MG draft genome sequence would provide genetics and genomic basis in understanding and improving its application to controlling PWD.

\section{Acknowledgments}

This work was supported by a grant from the National Institute of Forest Science (Project No. FE0702-2016-022016).

\section{References}

Agrawal, S., Adholeya, A. and Deshmukh, S. K. 2016. The Pharmacological potential of non-ribosomal peptides from marine sponge and tunicates. Front. Pharmacol. 7:333.

Altschul, S. F., Gish, W., Miller, W., Myers, E. W. and Lipman, D. J. 1990. Basic local alignment search tool. J. Mol. Biol. 215:403-410.

Aziz, R. K., Bartels, D., Best, A. A., DeJongh, M., Disz, T., Edwards, R. A., Formsma, K., Gerdes, S., Glass, E. M., Kubal, M., Meyer, F., Olsen, G. J., Olson, R., Osterman, A. L., Overbeek, R. A., McNeil, L. K., Paarmann, D., Paczian, T., Parrello, B., Pusch, G. D., Reich, C., Stevens, R., Vassieva, O., Vonstein, V., Wilke, A. and Zagnitko, O. 2008. The RAST server: rapid annotations using subsystems technology. BMC Genomics 9:75.

Bao, W., Zhou, Y., Jiang, J., Xu, Z., Hou, L. and Leung, F. C. 2015. Complete genome sequence of Raoultella ornithinolytica strain S12, a lignin-degrading bacterium isolated from forest soil. Genome Announc. 3:e00104-15.

Chin, C. S., Alexander, D. H., Marks, P., Klammer, A. A., Drake, J., Heiner, C., Clum, A., Copeland, A., Huddleston, J.,
Eichler, E. E., Turner, S. W. and Korlach, J. 2013. Nonhybrid, finished microbial genome assemblies from long-read SMRT sequencing data. Nat. Methods 10:563-569.

Choi, H. I., Park, J. Y., Shin, S. C. and Park, I. K. 2006. Nematicidal activity of medicinal plant extracts and two cinnamates isolated from Kaempferia galanga L. (Proh Hom) against the pine wood nematode, Bursaphelenchus xylophilus. Nematology 8:359-365.

Eljounaidi, K., Lee, S. K. and Bae, H. 2016. Bacterial endophytes as potential biocontrol agents of vascular wilt diseases - review and future prospects. Biol. Control 103:62-68.

Fan, W., Idnurm, A., Breger, J., Mylonakis, E. and Heitman, J. 2007. Eca1, a sarcoplasmic/endoplasmic reticulum $\mathrm{Ca}^{2+}$ ATPase, is involved in stress tolerance and virulence in Cryptococcus neoformans. Infect. Immun. 75:3394-3405.

Guo, Q., Du, G., Qi, H., Zhang, Y., Yue, T., Wang, J. and Li, R. 2017. A nematicidal tannin from Punicagranatum L. rind and its physiological effect on pine wood nematode (Bursaphelenchus xylophilus). Pestic. Biochem. Physiol. 135:64-68.

He, F. 2011. E. coli genomic DNA extraction. Bio-protocol Bio101:e97.

Ikeda, H., Nonomiya, T., Usami, M., Ohta, T. and Ōmura, S. 1999. Organization of the biosynthetic gene cluster for the polyketide anthelmintic macrolide avermectin in Streptomyces avermitilis. Proc. Natl. Acad. Sci. U.S.A. 96:9509-9514.

Jayaraj, R., Megha, P. and Sreedev, P. 2016. Organochlorine pesticides, their toxic effects on living organisms and their fate in the environment. Interdiscip. Toxicol. 9:90-100.

Khan, C. E., Jeon, J., Rim, O. S., Park, Y.-H., Lee, K. S. and Bae, H. 2017. Composition, diversity and bioactivity of culturable bacterial endophytes in mountain-cultivated ginseng in Korea. Sci. Rep. 7:10098.

Kim, J., Lee, S. M. and Park, C. G. 2016. Bursaphelenchus xylophilus is killed by homologues of 2-(1-undecyloxy)-1-ethanol. Sci. Rep. 6:29300.

Lee, S. M., Chung, Y. J., Moon, Y. S., Lee, S. G., Lee, D. W., Choo, H. Y. and Lee, C. K. 2003. Insecticidal activity and fumigation conditions of several insecticides against Japanese pine sawyer (Monochamus alternatus) larvae. J. Korean For. Soc. 92:191-198. 
Leung, F., Liu, L. and Jiang, J. 2016. Raoultella ornithinolytica strain A14 chromosome, complete genome deposited in National Centre for Biotechnology Information genome database. Deposited on: 9th August 2016. URL https://www.ncbi. nlm.nih.gov/nuccore/CP008886.1/ [9 February 2018].

Maniatis, T., Fritsch, E. F. and Sambrook, J. 1982. Molecular cloning a laboratory manual. 4th ed. Cold Spring Harbor Laboratory, Cold Springs Harbor, NY, USA.

Mota, M. and Vieira, P. 2008. Pine wilt disease: a worldwide threat to forest ecosystems. Springer, Heidelberg, Germany.

Overmars, L., van Hijum, S. A., Siezen, R. J. and Francke, C. 2015. CiVi: circular genome visualization with unique features to analyze sequence elements. Bioinformatics 31:28672869.

Paul, N. C., Ji, S. H., Deng, J. X. and Yu, S. H. 2013. Assemblages of endophytic bacteria in chili pepper (Capsicum annuum L.) and their antifungal activity against phytopathogens in vitro. Plant Omics 6:441-448.

Shin, S. H., Um, Y., Beak, J. H., Kim, S., Lee, S., Oh, M.-K., Kim, Y.-R., Lee, J. and Yang, K.-S. 2013. Complete genome sequence of Raoultella ornithinolytica strain B6, a 2,3-butanediol-producing bacterium isolated from oil-contaminated soil. Genome Announc. 1:e00395-13.

Takai, K., Suzuki, T. and Kawazu, K. 2003. Development and preventative effect against pine wilt disease of a novel liquid formulation of emamectin benzoate. Pest. Manag. Sci. 59:365-370.

Tatusova, T., DiCuccio, M., Badretdin, A., Chetvernin, V., Nawrocki, E. P., Zaslavsky, L., Lomsadze, A., Pruitt, K. D., Borodovsky, M. and Ostell, J. 2016. NCBI prokaryotic genome annotation pipeline. Nucl. Acids Res. 44:6614-6624.

Weber, T., Blin, K., Duddela, S., Krug, D., Kim, H. U., Bruccoleri, R., Lee, S. Y., Fischbach, M. A., Müller, R., Wohlleben, W., Breitling, R., Takano, E. and Medema, M. H. 2015. antiSMASH 3.0-a comprehensive resource for the genome mining of biosynthetic gene clusters. Nucl. Acids Res. 43:W237-W243.

Xia, Y., Xie, S., Ma, X., Wu, H., Wang, X. and Gao, X. 2011. The purL gene of Bacillus subtilis is associated with nematicidal activity. FEMS Microbiol. Lett. 322:99-107.

Zheng, Z., Zheng, J., Liu, H., Peng, D. and Sun, M. 2016a. Complete genome sequence of Fictibacillus phosphorivorans G25-29, a strain toxic to nematodes, J. Biotechnol. 239:2022.

Zheng, Z., Zheng, J., Zhang, Z., Peng, D. and Sun, M. 2016 b. Nematicidal spore-forming Bacilli share similar virulence factors and mechanisms. Sci. Rep. 6:31341. 